\title{
Double Parton Scattering Singularity in One-Loop Integrals
}

\author{
Jonathan R. Gaunt ${ }^{* \dagger}$ \\ University of Cambridge \\ E-mail: gauntdhep.phy.cam.ac.uk
}

\begin{abstract}
We discuss the double parton scattering (DPS) singularity, which is a specific type of Landau singularity that can occur in certain one-loop graphs in theories with massless particles. We sketch the derivation of a simple analytic formula for the DPS singular part of an arbitrary one-loop diagram. This is used to explain some results for DPS singularities in four- and six-point integrals that have been obtained using traditional loop integration techniques. As part of this we explain why the specific MHV and NMHV six-photon amplitudes often studied by the NLO multileg community are not divergent at the DPS singular point, and point out that whilst all NMHV six-photon amplitudes are always finite, certain MHV amplitudes do contain a logarithmic DPS divergence.
\end{abstract}

10th International Symposium on Radiative Corrections (Applications of Quantum Field Theory to Phenomenology) - Radcor2011

September 26-30, 2011

Mamallapuram, India

\footnotetext{
* Speaker.

${ }^{\dagger}$ This report summarises work performed in collaboration with W. J. Stirling. JG is supported by the UK Science and Technology Facilities Council.
} 


\section{Introduction}

One-loop integrals can become singular when the four-dimensional real hypercontour over which the integration is performed becomes pinched by two (or more) poles associated with denominator factors in the integrand. Such pinch singularities in a Feynman integral are known as Landau singularities. The locations of the Landau singularities in a one-loop Feynman graph are entirely determined by the propagator denominator factors, and so are independent of the nature of the particles in the graph. On the other hand, the behaviour of the integral at the singular points does depend on the nature of the particles in the graph via the numerator factor of the Feynman integrand - if the numerator vanishes at a singular point, the integral will be less singular than expected there, or even finite.

In this proceedings contribution we discuss a particular type of Landau singularity known as the double parton scattering (DPS) singularity, that was first discussed in [四]. This singularity occurs in one-loop diagrams with the structure of figure W(a), where two on-shell massless particles each split into two massless particles, and the four resulting particles interact in two separate processes, each producing a set of particles with positive invariant mass. It only occurs when the total transverse momentum of each set of outgoing particles is zero $\left(\mathbf{Q}_{2}=0\right)$, and is associated with the four intermediate particles becoming on-shell and collinear with the initial state particles. [Note that in this proceedings contribution we use bold letters to denote two-component transverse momentum vectors].

There are two reasons why it is interesting to study the behaviour of one-loop integrals around their DPS singular point(s). First, it has been observed that certain one-loop Standard Model (SM) multileg amplitudes are less singular than perhaps expected near points corresponding to a DPS singularity for a subset of the contributing Feynman graphs [《, [], 田]. A physical explanation for

(a)

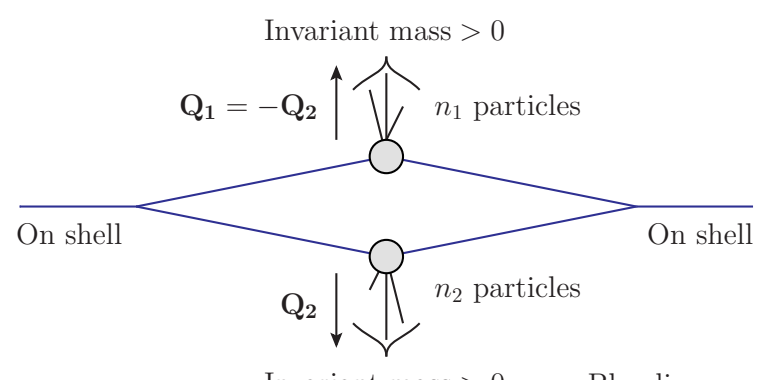

Invariant mass $>0$ (b)

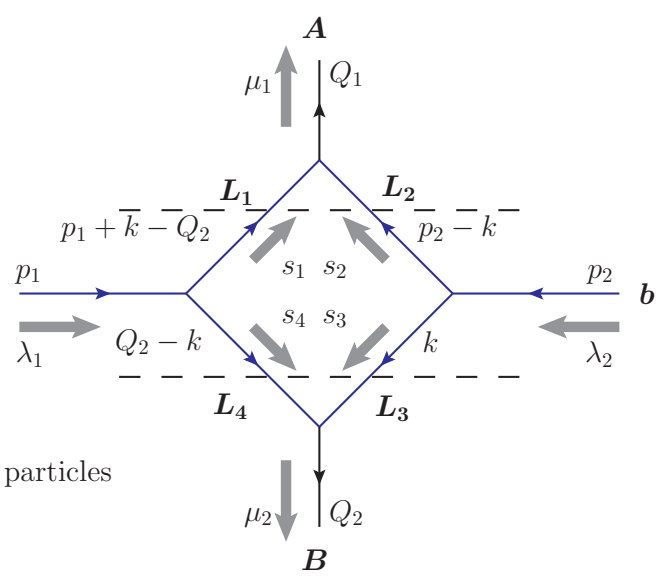

Figure 1: (a) The generic structure that a one loop graph must have in order for it to possess a DPS singularity. Each grey blob represents some arbitrary tree-level structure. (b) The crossed box topology, with annotations that demonstrate our labelling conventions for the particle names, helicity and momenta. The particle names are written in bold in this figure, whilst the helicity labels are accompanied by grey arrows. The arrows on the lines merely indicate the direction of momentum flow, and do not necessarily signify a fermion line. We also include the two Cutkosky cuts of this box as dashed lines. 
this behaviour is needed. Second, an understanding of the nature of the DPS singularity in an arbitrary one-loop graph is interesting from the point of view of double parton scattering theory, and can be used to show that there are theoretical flaws in the framework for calculating protonproton DPS cross sections described in [ [ $[$, 6 ]. In this contribution we focus on the first point, and refer the reader interested in the second to [ $[$, 四]. This contribution is a summary of part of the paper [8]

\section{DPS Singularity in One-Loop Integrals}

With no numerator structure in the integrand (i.e. scalar internal and external particles), the DPS singularity corresponds to an actual divergence in the loop integral (when the number of dimensions is equal to 4). For the simplest graph that can contain a DPS singularity - the 'crossed box' diagram of figure $\mathbb{W}(\mathrm{b})$ - an explicit calculation of the scalar integral reveals a divergence like $1 / \mathbf{Q}_{2}^{2}$, and a similar power divergence is expected (and indeed turns out to be observed) for more general scalar loops. Naively, one might expect the behaviour of SM loops at the DPS singular point to be similar - they can only be less divergent if the numerator happens to vanish at the singular point, as mentioned in the introduction.

However, explicit results for $n$-point amplitudes (with $n$ small) that have been obtained using conventional loop integral techniques suggest that such a strong divergence is not present in SM loops initiated by two $g / \gamma \rightarrow q \bar{q}$ splittings (we'll refer to these loops as 'SM $q \bar{q} q \bar{q}$ loops'). Examples of four-point amplitudes of this kind for which we have explicit (analytic) results are the $g g \rightarrow H H$ and $g g \rightarrow Z Z$ amplitudes via massless quarks, with the analytic results being presented (for arbitrary quark mass) in [Q] and [ㅁ] ]. From these results it is possible to extract the leading low $\mathbf{Q}_{2}$ behaviour of the $g g \rightarrow H H$ and $g g \rightarrow Z Z$ crossed boxes via massless quarks [ $[\mathbb{]}$. One finds that neither of these graphs diverges as $\mathbf{Q}_{\mathbf{2}} \rightarrow 0$ more strongly than a logarithm of $\mathbf{Q}_{\mathbf{2}}$, regardless of the configuration of external helicity in each case. In fact, neither graph diverges at all unless the helicity of the initial state gluons is the same, and the $g g \rightarrow Z Z$ graph does not diverge unless the helicities of the final state $Z$ bosons are ++ or -- .

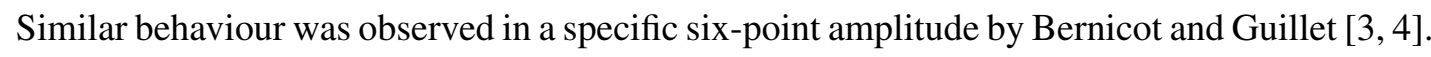
The process that was studied by these authors was $2 \gamma \rightarrow 4 \gamma$ via massless quarks, analytical results for which are available in [ㅁ], [2]. Labelling the six external legs as '123456', and taking legs 1 and 4 as incoming with the rest outgoing, Bernicot and Guillet made plots of a particular MHV and a particular NMHV amplitude close to the kinematic point $\mathbf{k}_{\mathbf{t}} \equiv \mathbf{p}_{3}+\mathbf{p}_{5}=0$ corresponding to a DPS singularity for four of the contributing Feynman graphs. The helicity configuration for the MHV amplitude examined was -++-++ (where the helicity labels here are defined with respect to incoming momenta), and that for the NMHV amplitude was ---+++ (these two helicity configurations are often studied by the NLO multileg community). From their plots they concluded that both the MHV and the NMHV amplitudes are finite at $\mathbf{k}_{\mathbf{t}}=0$, contrary to the naive expectation of a power divergence at this point [田].

Therefore, there are two important questions that we need to answer. First, what is the physical mechanism that prevents the DPS divergence in any of these SM $q \bar{q} q \bar{q}$ graphs being stronger than a logarithm of $\mathbf{Q}_{2}$ ? Second, why are only some of these SM graphs divergent, whilst others are finite? To answer these questions an analytical study of the DPS divergence in one loop integrals is 
required, involving the derivation of an analytic formula for the 'DPS divergent part' of an arbitrary one-loop integral. The 'DPS divergent part' of a one-loop integral is an expression for the part of the integral in which the loop particles are almost on-shell and collinear, that is valid in the limit in which external transverse momenta are small.

In [8], we derived an analytic expression for the DPS divergent part of the arbitrary crossed box graph in figure $\square(b)$, before generalising the result to the case in which the number of external particles is arbitrary. Here we briefly summarise this derivation. We begin with an expression for the loop integral $L$ :

$$
L=\int d^{d} k \frac{\mathscr{N}}{\left[k^{2}+i \varepsilon\right]\left[\left(k-Q_{2}\right)^{2}+i \varepsilon\right]\left[\left(p_{1}+k-Q_{2}\right)^{2}+i \varepsilon\right]\left[\left(p_{2}-k\right)^{2}+i \varepsilon\right]}
$$

The propagator denominator factors are written out explicitly in this expression, since they are universal to all crossed boxes, whilst the numerator $\mathscr{N}$ is kept general. $L$ is defined such that $\mathscr{N}$ is stripped of overall factors such as coupling constants, colour factors, and factors of $i$ (including the overall factor of $-i$ that always has to be inserted in the computation of an amplitude).

We decompose all four-vectors in (2. (1) terms of a light-cone basis defined using the incoming vectors $p_{1}$ and $p_{2}$ as the light-like basis vectors. For an arbitrary four-vector $A$ the decomposition is written as follows:

$$
A=\left(A^{+} p_{1}+A^{-} p_{2}\right) / \sqrt{p_{1} \cdot p_{2}}+\mathbf{A}
$$

After this, the integrations over the plus and minus components of the loop variable $k$ are explicitly performed one after the other using contour methods. At each stage in this procedure we drop terms that are negligible in the region around the DPS singularity $\left|k^{-}-Q_{2}^{-}\right|,\left|k^{+}\right|,|\mathbf{k}|,\left|\mathbf{k}-\mathbf{Q}_{\mathbf{2}}\right| \ll$ $Q_{i}^{+}, Q_{i}^{-}, i=1,2$. In addition to this we make use of various approximations that are only valid in this region and that facilitate the calculation of the integrals (for full details of these approximations, see $[\mathbb{[}]$ ). This procedure yields the following compact expression for the DPS divergence in the crossed box:

$$
L_{D P S} \simeq \frac{(2 \pi i)^{2}}{2 s} \int_{|\mathbf{k}| \ll Q_{i}^{+}, Q_{i}^{-}} \frac{\left.d^{d-2} \mathbf{k} \mathscr{N}\right|_{k^{-}=Q_{2}^{-}, k^{+}=0}}{\left(\mathbf{k}-\mathbf{Q}_{\mathbf{2}}\right)^{2} \mathbf{k}^{2}}
$$

One can go further than this if one notices that the DPS divergence (2.31) occurs entirely in the real part of the loop integral $L$, which corresponds to the imaginary part of the box amplitude according to the way in which we have defined $L$. Then, the DPS divergent part of a crossed box can be found as the sum of the Cutkosky cuts of the box in the limit in which external transverse momenta are small and internal particles are almost on shell. There are two Cutkosky cuts of the box (shown in figure $\mathbb{W}(\mathrm{b})$ ), and they give identical contributions in the relevant limit, so in what follows it suffices to consider just one of the cut diagrams (and multiply the answer by 2).

Having separated out one of the 'hard scatterings' in the DPS divergent part of the crossed box using the cut, the other may be separated out using completeness relations that are approximately valid in the small virtuality limit that we are considering (this is schematically depicted in figure D). We then obtain $L_{D P S}$ as an integral over four factors, each of which corresponds to one of the 


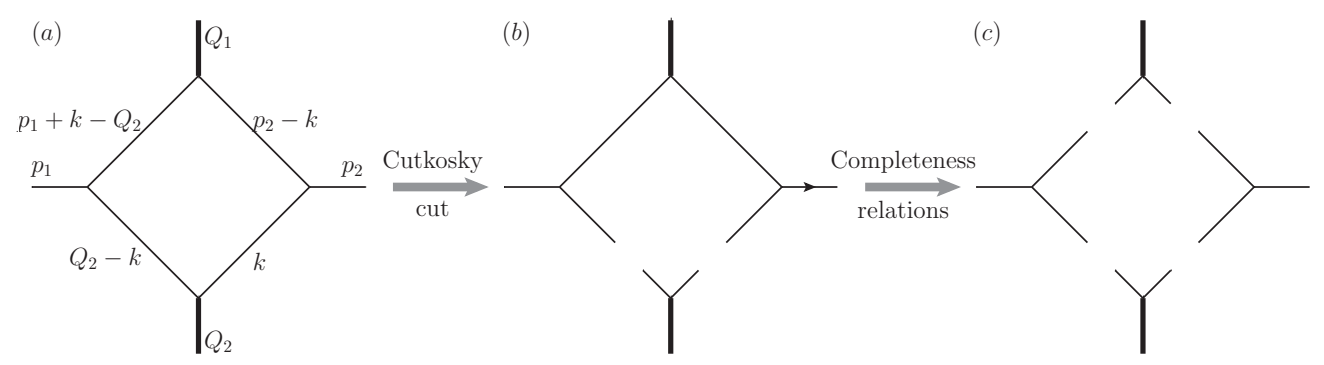

Figure 2: Decomposition of the box integrand.

broken pieces of the crossed box drawn in figure $\mathbb{\nabla}(\mathrm{c})$ :

$$
\begin{aligned}
L_{D P S}\left(\lambda_{1} \lambda_{2} \mu_{1} \mu_{2}\right) & =\sum_{s_{i}, L_{i}} \int d^{d} k \delta\left(k^{2}\right) \delta\left(\left(k-Q_{2}\right)^{2}\right)\left(\sqrt{Q_{1}^{2} / Q_{2}^{2}}\right) \Phi_{b \rightarrow L_{2} L_{3}}^{\lambda_{2} \rightarrow s_{2} s_{3}}\left(p_{2} ; p_{2}-k, k\right) \\
& \times \Phi_{a \rightarrow L_{1} L_{4}}^{\lambda_{1} \rightarrow s_{1} s_{4}}\left(p_{1} ; p_{1}+k-Q_{2}, Q_{2}-k\right) \mathscr{M}_{L_{3} L_{4} \rightarrow B}^{s_{3} s_{4} \rightarrow \mu_{2}}\left(k, Q_{2}-k ; Q_{2}\right) \\
& \times \mathscr{M}_{L_{1} L_{2} \rightarrow A}^{s_{1} s_{2} \rightarrow \mu_{1}}\left(p_{1}+k-Q_{2}, p_{2}-k ; Q_{1}\right)
\end{aligned}
$$

$\Phi_{a \rightarrow L_{1} L_{4}}$ is the lowest order light-cone wavefunction (LCWF) to produce a $L_{1} L_{4}$ pair from the particle $a$, whilst $\mathscr{M}_{L_{1} L_{2} \rightarrow A}^{s_{1} s_{2} \rightarrow \mu_{1}}$ is the usual lowest order matrix element for the process $L_{1} L_{2} \rightarrow A$. Each LCWF in the above expression contains a matrix element corresponding to one of the initial state splittings in figure $\nabla(c)$, divided by the denominator of the propagator closest to this splitting vertex in figure $\mathbb{Z ( b )}$. For the purposes of evaluating the leading low $\mathbf{Q}_{\mathbf{2}}$ divergence, the matrix elements in (2.4) can be evaluated with the transverse momentum and virtuality of the initial state particles set to zero, since the matrix elements are non-singular in this limit.

Having obtained the formula (2.4) for the DPS divergence in a crossed box diagram, the generalisation of the formula to the arbitrary loop in figure $\mathbb{W}(\mathrm{a})$ is clear. All one needs to do is to replace the two $2 \rightarrow 1$ tree level matrix elements in (2.4) by expressions for the relevant $2 \rightarrow n_{1}$ and $2 \rightarrow n_{2}$ tree graphs.

Let us now consider the behaviour of the LCWFs in (2.4). These can in general be decomposed into two factors, one of which depends on the light-cone momentum fraction of one of the daughter partons with respect to the parent parton, and the other of which depends on the transverse momentum of that daughter parton with respect to the parent:

$$
\Phi_{a \rightarrow b c}^{\lambda \rightarrow s_{1} s_{2}}(p ; k, p-k)=X_{a \rightarrow b c}^{\lambda \rightarrow s_{1} s_{2}}(x) K_{a \rightarrow b c}^{\lambda \rightarrow s_{1} s_{2}}(\mathbf{k})
$$

The factor $X$ can be thought of as the square root of a helicity dependent splitting function. The transverse momentum dependent factor $K$ contains a $1 / \mathbf{k}^{2}$ factor from the propagator denominator absorbed in the LCWF, multiplied by the $\mathbf{k}$ dependent part of the $a \rightarrow b c$ matrix element.

In the case of scalar $\phi^{3}$ theory, the splitting matrix element is just a constant, so $K(\mathbf{k})$ in this theory $\propto 1 / \mathbf{k}^{2}$. Then, for an arbitrary loop, we obtain a power divergence at the DPS singular point when $d=4$ (as we mentioned earlier):

$$
L_{D P S, \phi^{3}} \sim \int d^{d-2} \mathbf{k} K(\mathbf{k}) K\left(\mathbf{Q}_{\mathbf{2}}-\mathbf{k}\right) \propto \int \frac{d^{d-2} \mathbf{k}}{\mathbf{k}^{2}\left(\mathbf{Q}_{\mathbf{2}}-\mathbf{k}\right)^{2}} \propto \frac{1}{\mathbf{Q}_{2}^{2}} \text { when } d=4 .
$$


On the other hand, for the $g / \gamma \rightarrow q \bar{q}$ SM splittings, the matrix element comes out as being proportional to $\mathbf{k}$. This is associated with the fact that these processes do not conserve $J_{z}$, and so must be forbidden, in the collinear limit $\mathbf{k} \rightarrow 0$ (the initial state has magnitude of $J_{z}=1$, whilst the final state must have $J_{z}=0$ in the collinear limit due to helicity conservation). For a SM $q \bar{q} q \bar{q}$ loop, we therefore have, schematically:

$$
L_{D P S, S M} \sim \int d^{d-2} \mathbf{k} K(\mathbf{k}) K\left(\mathbf{Q}_{\mathbf{2}}-\mathbf{k}\right) \sim \int \frac{d^{d-2} \mathbf{k} \mathbf{k}^{i}\left(\mathbf{Q}_{\mathbf{2}}-\mathbf{k}\right)^{j} T^{i j}}{\mathbf{k}^{2}\left(\mathbf{Q}_{\mathbf{2}}-\mathbf{k}\right)^{2}} \sim \log \left(\mathbf{Q}_{\mathbf{2}}{ }^{2}\right)
$$

where $T^{i j}$ is some $\mathbf{k}$-independent matrix.

Thus, the DPS divergence in a SM $q \bar{q} q \bar{q}$ loop cannot be stronger than a logarithm of $\mathbf{Q}_{\mathbf{2}}$. The underlying cause of this is $J_{z}$ nonconservation in $g / \gamma \rightarrow q \bar{q}$ massless particle splittings in the collinear limit.

The schematic estimate (․ㄱ) does not take account of the detailed structure of the LCWFs and the matrix elements $\mathscr{M}$, and so only gives an upper bound on the degree of divergence in a SM $q \bar{q} q \bar{q}$ integral (this is why we have written 'cannot be stronger than', rather than 'is', in the preceding paragraph). There can be additional suppression from either the LCWFs or the matrix elements (or both) at the DPS singular point, that causes the DPS divergence to disappear entirely. This is what is occurring in the four- and six-point amplitudes mentioned earlier that are finite at the DPS singular point.

For any SM $q \bar{q} q \bar{q}$ loop graph, there is an additional suppression occurring in the LCWFs when the helicities of the initial state gluons or photons are different. In this situation, it turns out that the product of light cone wavefunctions vanishes upon integration over $\mathbf{k}$ when $\mathbf{Q}_{\mathbf{2}}=0$, such that the graph is finite at $\mathbf{Q}_{\mathbf{2}}=0$. The underlying physical cause of this phenomenon can be exposed by sketching the helicities of the initial state and the $q \bar{q} q \bar{q}$ intermediate state that is long-lived in the collinear limit:

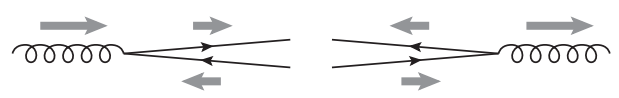

The magnitude of $J_{z}$ of the $g g / \gamma \gamma$ initial state is 2, whilst that of the $q \bar{q} q \bar{q}$ intermediate state must be 0 in the collinear limit due to helicity conservation. Thus there is an issue of total $J_{z}$ conservation in this case aside from local $J_{z}$ conservation at each splitting vertex - this is the source of the additional suppression. Note that this is the reason why the NMHV six-photon amplitude studied by Bernicot and Guillet is finite at the DPS singular point.

For the $g g \rightarrow Z Z$ crossed box, there is a suppression coming from the matrix element factors when the helicities of the final state particles are not ++ or -- . In this case there is no configuration of internal helicity in the loop which simultaneously ensures helicity conservation at every external vertex, and conserves $J_{z}$ in both $q \bar{q} \rightarrow Z$ processes in the collinear limit. This causes at least one of the $q \bar{q} \rightarrow Z$ matrix elements in the DPS divergence formula (‥4) to vanish for all internal helicity configurations allowed by the LCWF factors.

The finiteness of the $2 \gamma \rightarrow 4 \gamma$ MHV amplitude studied by Bernicot and Guillet at $\mathbf{k}_{\mathbf{t}}=0$ may similarly be explained by a suppression in the matrix element factors. Here, the DPS divergence does not vanish on a graph-by-graph basis (as it does for the NMHV amplitude) - it only vanishes when we sum up the four graphs that contain a DPS singularity at $\mathbf{k}_{\mathbf{t}}=0$. The DPS divergence in 
the sum of the four graphs may be computed by using (․․), with full $q \bar{q} \rightarrow \gamma \gamma$ tree-level matrix elements (each one being a sum of two tree graphs) replacing the matrix elements given there. For the MHV $2 \gamma \rightarrow 4 \gamma$ amplitude studied, the final-state photons have the same helicity in both of these $q \bar{q} \rightarrow \gamma \gamma$ matrix element factors. The matrix element factors are therefore zero according to the MHV rules for QED [[13], and the DPS divergence is nullified.

Using our approach one may make statements about the degree of DPS divergence in other $2 \gamma \rightarrow 4 \gamma$ helicity amplitudes without performing further calculations. For example, we can say that no NMHV six-photon amplitude can ever contain a DPS divergence - however one distributes the helicities, one always ends up either with the initial state photons having opposite helicities, or with one of the pairs of the final state photons having the same helicity. On the other hand there are MHV amplitudes that have a logarithmic DPS divergence - for example the +--+++ amplitude.

\section{Conclusions}

In this contribution we have outlined the derivation of a compact analytic expression for the DPS divergence in an arbitrary one-loop diagram. The expression essentially consists of an integral of two light-cone wavefunctions and two hard matrix elements over the transverse components of the loop momentum. We used this expression to show that no SM loop initiated by two $g / \gamma \rightarrow q \bar{q}$ splittings ('SM $q \bar{q} q \bar{q}$ loop') can have a DPS divergence worse than a logarithm of $\mathbf{Q}_{\mathbf{2}}$, and explained that this behaviour is associated with $J_{z}$ nonconservation in, and the consequent suppression of, $g / \gamma \rightarrow q \bar{q}$ SM splittings in the collinear limit. We observed that the DPS divergence in SM $q \bar{q} q \bar{q}$ loops may disappear if there is some additional suppression at the DPS singular point coming from either the light-cone wavefunctions or the hard matrix elements (or both). The $g g \rightarrow A B$ box amplitude with unequal initial-state gluon helicities (with $A, B$ arbitrary) and the NMHV - -+++ six-photon amplitude are examples of amplitudes which are finite due to a suppression in the light-cone wavefunctions. On the other hand, the $g g \rightarrow Z Z$ box amplitude with final state helicities not equal to ++ or -- , and the MHV -++-++ six-photon amplitude, are examples of amplitudes which are finite due to a suppression in the matrix element factors. Finally, we made use of our approach to point out that whilst no NMHV six-photon amplitude contains a DPS divergence, there are MHV amplitudes that do contain logarithmic DPS divergences.

\section{References}

[1] Z. Nagy and D. E. Soper, Numerical integration of one-loop Feynman diagrams for N-photon amplitudes, Phys.Rev. D74 (2006) 093006, [hep-ph/0610028].

[2] L. D. Ninh, One-Loop Yukawa Corrections to the Process $p p \rightarrow b \bar{b} H$ in the Standard Model at the LHC: Landau Singularities, arXiv:0810.4078.

[3] C. Bernicot, The six-photon amplitude, arXiv:0804.1315.

[4] NLO Multileg Working Group Collaboration, Z. Bern et. al., The NLO multileg working group: Summary report, arXiv:0803.0494.

[5] G. M. Zinovev, A. M. Snigirev, and V. P. Shelest, Equations for many parton distributions in quantum chromodynamics, Theor. Math. Phys. 51 (1982) 523-528. 
[6] A. M. Snigirev, Double parton distributions in the leading logarithm approximation of perturbative QCD, Phys. Rev. D68 (2003) 114012, [hep-ph/0304172].

[7] J. R. Gaunt, Double Parton Splitting Diagrams and Interference and Correlation Effects in Double Parton Scattering, arxiv:1110.1536.

[8] J. R. Gaunt and W. J. Stirling, Double Parton Scattering Singularity in One-Loop Integrals, JHEP 1106 (2011) 048, [arXiv:1103.1888].

[9] E. Glover and J. van der Bij, Higgs boson pair production via gluon fusion, Nucl.Phys. B309 (1988) 282.

[10] E. Glover and J. van der Bij, Z boson pair production via gluon fusion, Nucl.Phys. B321 (1989) 561.

[11] T. Binoth, G. Heinrich, T. Gehrmann, and P. Mastrolia, Six-Photon Amplitudes, Phys.Lett. B649 (2007) 422-426, [hep-ph/0703311].

[12] C. Bernicot and J.-P. Guillet, Six-Photon Amplitudes in Scalar QED, JHEP 0801 (2008) 059, [arXiv:071].4713].

[13] K. J. Ozeren and W. J. Stirling, MHV techniques for QED processes, JHEP 11 (2005) 016, [hep-th/0509063]. 